\title{
(2) A Clinical Study of the "Water-Electrolyte Regulation Mechanism" in Chronic Congestive Heart Failure with Emphasis on Renin and Catecholamine
}

\author{
Hiroshi Saimyoj, M.D., Yoichi Seriu, M.D. and Masao Takayasu, M.D. \\ The Third Division, Department of Internal Medicine, Faculty of Medicine, \\ Kyoto University, Kyoto, Japan
}

In our laboratory, a series of clinical investigations on the endocrine factors in chronic congestive heart failure (CHF) has been conducted with special attention to the regulation mechanism. ${ }^{1-10)}$

Urinary aldosterone excretion was often high in patients with right- and bothsided heart failure, and showed a positive correlation with the degree of edema. In cases successfully treated with bed rest and/or digitalis, aldosterone was reduced to and remained within normal limits. In cases requiring continued use of diuretics in addition to rest and digitalis, aldosterone was reduced temporarily but then rose again. In the latter cases, CHF persisted and aldosterone played a physiological role in maintaining homeostasis in response to the continuous loss of sodium and water induced by forced diuresis.

Urinary excretion of catecholamines (CA), especially norepinephrine (NE) increased progressively as the functional class (N. Y. H. A.) proceeded. In cases treated successfully with rest and/or digitalis, the increased NE returned to normal. In cases resistant to treatment, the high excretion rate of $\mathrm{NE}$ persisted. When an adrenolytic drug was administered in cases of hypertensive heart failure, the CHF was aggravated. These results suggest that the sympatho-adrenomedullary system contributes to the support of the circulatory function in CHF.

In response to water-loading $(10 \mathrm{ml} / \mathrm{kg}$ by mouth), the plasma ADH concentration was reduced in 12 of the 15 tests, and water diuresis occurred in 20 out of 27 tests. In one patient, plasma ADH was determined repeatedly and showed a positive correlation with serum osmolality, and in the 27 tests, there was a significant positive correlation between peripheral venous pressure and serum osmolality. These results suggest that an elevation in venous pressure causes an increase in extravasation of body fluid and a decrease in venous return with an increased osmotic pressure of venous blood, and that these changes in body fluids may stimulate the secretion of $\mathrm{ADH}$. Even in $\mathrm{CHF}, \mathrm{ADH}$ seems to act homeostatically together with aldosterone in adjusting the blood volume to maintain the venous pressure and hence the circulatory function.

The authors treated two patients with severe CHF, in whom hyponatremia, 
hyposmolality and marked hypovolemia were induced by continuous administration of potent diuretics, and the secretion of $\mathrm{ADH}$ increased while that of aldosterone did not. It was thought that it was crucial for existence under these circumstances to maintain the circulating blood volume rather than the osmolality.

The present study was, therefore, undertaken to investigate the effects of furosemide-induced acute isotonic diuresis on the humoral factors in relation to plasma volume (PV) changes in $\mathrm{CHF}$.

\section{Subjects and Methods}

Twenty-eight tests were performed on 5 normal volunteers and 21 inpatients with CHF. After 14 hours of deprivation of water and food, furosemide $(40 \mathrm{mg})$ was given by mouth with $50-100 \mathrm{ml}$ of water. Blood samples were drawn from the antecubital vein. Plasma renin activity (PRA) was determined at 15, 30, 60, 120, 180 and 240 minutes and $\mathrm{PV}$, at 1,2 and 4 hours, after furosemide ingestion. Plasma $\mathrm{ADH}$ was determined at the time of expected maximum diuresis. The analytical methods used were: PRA by radioimmunoassay of angiotensin I using ${ }^{125} \mathrm{I}$ in cases 1-8, and by Pickens' method in cases 9-14; urinary CA by Euler-Lishajko's method; urinary aldosterone by Kliman-Peterson's double isotope derivative assay; plasma ADH by bioassay (Yamane-Kunishige's method); and PV by ${ }^{131}$ I-RIHSA.

\section{Results and Discussion}

The main results are shown in Table $I$. In cases without edema, the PV decreased and epinephrine (E), PRA, aldosterone and ADH increased, in response to the profuse diuresis. In edematous patients, despite a comparable diuresis, the PV did not decrease and the secretion of those hormones was not stimulated. In edematous patients, any deficiency in the PV induced by diuretics can be replenished from the expanded interstitial fluid depot, so that only small changes, if any, occur in the PV and no appreciable increases occur in $\mathrm{ADH}$, aldosterone, CA or PRA. This replenishment is more difficult in subjects without edema, and a significant PV depletion occurs, which stimulates the secretion of the humoral factors. During the acute furosemide test, there were no appreciable changes in serum sodium or potassium levels. Thus it is probable that under the test conditions, a decrease in PV is the adequate stimulus for the regulation mechanism, rather than the loss of sodium itself, the loss of extracellular fluid or changes in serum osmolality, sodium or potassium concentrations. There are several reports that point to the primary role of $\mathrm{PV}$ in the effects of potent diuretics on PRA and aldosterone. ${ }^{8-15)}$

It is accepted that $\mathrm{CA}$ stimulates the release of $\mathrm{ADH}$, and that vasopressin and vasotocin suppress the release of renin. CA and renin stimulate the secretion of each other. Thus it is probable that renin acts as a mediator among those factors that constitute the regulation mechanism. So far there have been no clinical reports 
TABLE I THE RESPONSE OF URINE VOLUME, PLASMA YOLUME, PLASMA RENIN ACTIVITY, EPINEPHRINE, NOREP INEPHRINE,
ALDOSTERONE AND ANT TOIURETIC HORMONE TO ORAL AOMINISTRATION OF FUROSEMIDE (40Mg)

\begin{tabular}{|c|c|c|c|c|c|c|c|c|c|c|c|c|c|c|c|c|c|c|c|c|}
\hline CASE & \begin{tabular}{|c|c|}
$U \mathrm{~V}$ & $\mathrm{P}$ \\
$\mathrm{m} 1 / 30 \mathrm{~min}$ & $1 /$
\end{tabular} & $\begin{array}{l}P \vee \\
1 / m^{2}\end{array}$ & $\begin{array}{l}\text { PRA } \\
\mathrm{ng} / \mathrm{ml} \mathrm{hr}\end{array}$ & $\begin{array}{c}E \\
\mathrm{ng} / \min \end{array}$ & $\begin{array}{l}\text { NE } \\
\mathrm{ng} / \mathrm{min}\end{array}$ & $C A S E$ & & \begin{tabular}{|c|c|}
$U V$ & $P$ \\
$m 1 / 30 \min$ & 1.
\end{tabular} & $\mid \begin{array}{c}P \vee \\
1 / m^{2}\end{array}$ & PRA & $\begin{array}{c}E \\
\mathrm{ng} / \mathrm{min}\end{array}$ & $\begin{array}{l}N E \\
n g / \text { min }\end{array}$ & \begin{tabular}{|c|} 
ALDO \\
$\mu g / h n$
\end{tabular} & $\left|\begin{array}{l}A D H \\
\mu \mathrm{u} / \mathrm{m} 1\end{array}\right|$ & CASE & & $\begin{array}{c}U V \\
m 1 / 30 \mathrm{~min}\end{array}$ & $\begin{array}{l}P V \\
1 / m^{2}\end{array}$ & $\begin{array}{ll}\text { ALDO } \\
\mu g / \mathrm{hr}\end{array}$ & $\begin{array}{c}A 0 H \\
\mu \mathrm{u} / \mathrm{m}\end{array}$ \\
\hline $\begin{array}{l}1.58 . m_{i} \\
\text { I H o, Mi i } \\
\text { Pheochromo- } \\
\text { cytoma }\end{array}$ & 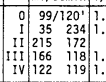 & $\begin{array}{l}1.88 \\
1.86 \\
1.71 \\
1.79\end{array}$ & \begin{tabular}{|c|}
1.59 \\
1.841 .78 \\
0.85 \\
1.42 \\
0.97 \\
\end{tabular} & 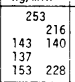 & 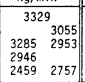 & \begin{tabular}{|ll} 
9. & \\
M S. f.
\end{tabular} & \begin{tabular}{r|}
0 \\
1 \\
II \\
111 \\
IV
\end{tabular} & \begin{tabular}{|cc|c|}
$49 / 131$ & 1 \\
115 & 470 \\
330 & 180 & 1 \\
515 & 20 \\
23 & 17 & 1 \\
\end{tabular} & \begin{tabular}{|l|l}
1.55 & 0 \\
1.33 & 1 \\
1.40 & \\
\end{tabular} & $\begin{array}{l}0.41 \\
1.55\end{array}$ & 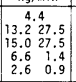 & 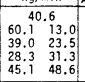 & $\begin{array}{l}0.92 \\
4.68 \\
0.23\end{array}$ & & $\begin{array}{l}17 . \\
\text { MS I, f. A I }\end{array}$ & $\mid$\begin{tabular}{r|}
0 \\
II \\
III \\
III
\end{tabular} & $\begin{array}{|cc|}69 / 30 \\
55 & 231 \\
362 & 283 \\
225 & 117\end{array}$ & \begin{tabular}{|l|}
1.89 \\
1.78 \\
1.80 \\
1.73
\end{tabular} & & $\begin{array}{r}<1.3 \\
1.7\end{array}$ \\
\hline $\begin{array}{l}\text { 2. } 28 . \mathrm{f} . \\
\text { A S D, M I }\end{array}$ & 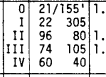 & $\begin{array}{l}1.30 \\
1.33 \\
1.27 \\
\end{array}$ & \begin{tabular}{|c|}
2.25 \\
2.80 \\
3.19 \\
9.43 \\
17.63 \\
12.2 \\
\end{tabular} & \begin{tabular}{|l|}
7.8 \\
11.3 \\
38.74 .4 \\
19.7 \\
19.626 .4 \\
30.627 .2 \\
\end{tabular} & 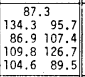 & 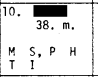 & $\begin{array}{r}0 \\
1 \\
11 \\
11 \\
11 \\
\text { IV }\end{array}$ & 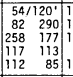 & $\begin{array}{l}1.96 \\
1.92 \\
1.86 \\
1.75 \\
1.75\end{array}$ & $\begin{array}{ll}0.82 \\
1.18 \\
0.21 \\
\end{array}$ & 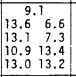 & \begin{tabular}{|l|l|}
36.2 & 36.2 \\
49.5 & 40.2 \\
37.8 & 53.4 \\
41.0 & 41.9 \\
29.6 & 33.6 \\
\end{tabular} & \begin{tabular}{|l|}
2.01 \\
4.05 \\
1.78 \\
\end{tabular} & & $\begin{array}{l}\text { 18. } \\
\text { 45. f. } \\
\text { Hypertension } \\
\text { Arterio- io } \\
\text { sclerosis }\end{array}$ & $\mid \begin{array}{r}0 \\
\text { I } \\
\text { II } \\
\text { II }\end{array}$ & 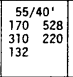 & $\begin{array}{l}1.61 \\
1.57 \\
1.31\end{array}$ & $\begin{array}{l}0.62 \\
1.67 \\
2.75\end{array}$ & $\begin{array}{l}2.2 \\
2.2\end{array}$ \\
\hline $\begin{array}{l}\text { 3. } 53 . \mathrm{f} \text {. } \\
\text { Angina } \\
\text { pectoris }\end{array}$ & \begin{tabular}{r|r|r}
0 & $138 / 115$ & 1 \\
II & 317 & 585 \\
II & 314 & 176 \\
III & 82 & 34 \\
IV & 62 & 34 \\
\end{tabular} & $\begin{array}{l}1.80 \\
1.56 \\
1.71 \\
1.43\end{array}$ & $\begin{array}{ll}0.21 & 0.27 \\
0.102 .02 \\
1.74 \\
1.77 \\
1.66\end{array}$ & \begin{tabular}{|c|}
5.6 \\
20.625 \\
19.9 \\
18.921 .4 \\
18.718 .0 \\
10.9
\end{tabular} & \begin{tabular}{|c|c|}
7.9 & 6.4 \\
8.4 & 6.0 \\
83.9 & 16.6 \\
23.9 & 17.2 \\
25.2 & \\
\end{tabular} & 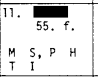 & $\begin{array}{r}0 \\
1 \\
\text { II } \\
\text { III } \\
\text { IV }\end{array}$ & 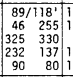 & $\mid$\begin{tabular}{l|l}
1.95 & 0 \\
1.76 & 0 \\
1.68 & 0 \\
1.57 & 0
\end{tabular} & $\begin{array}{ll}0.12 \\
0.16 \\
0.12 & \\
& \end{array}$ & 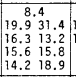 & 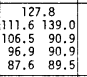 & $\begin{array}{l}0.34 \\
5.28 \\
2.66 \\
\end{array}$ & & $\begin{array}{l}\text { 19. } \\
62 . \mathrm{m} . \\
\text { A I }\end{array}$ & \begin{tabular}{r|}
0 \\
11 \\
IIt \\
IV \\
\end{tabular} & \begin{tabular}{|c|c|}
$307 / 130$ \\
42 & 122 \\
275 & 337 \\
277 & 184 \\
200 & 95 \\
\end{tabular} & $\begin{array}{l}2.01 \\
2.02 \\
1.91 \\
\end{array}$ & & $\begin{array}{r}1.0 \\
<0.8 \\
\end{array}$ \\
\hline $\begin{array}{l}\text { 4. } 66.7 . \\
\text { MS, A S }\end{array}$ & \begin{tabular}{r|rr|}
0 & $243 / 150^{1}$ \\
II & 337 & 545 \\
II & 400 & 302 \\
III & 171 & 125 \\
IV & 173 & 79 \\
\end{tabular} & $\begin{array}{l}1.88 \\
1.73 \\
1.53 \\
\end{array}$ & 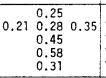 & \begin{tabular}{|c|c|} 
& 6.9 \\
11.3 & 9.0 \\
10.0 & 9.1 \\
11.4 & 7.1 \\
11.1 & 5.9 \\
\end{tabular} & 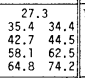 & $\begin{array}{ll}\text { 12. } & \\
& 42 . f . \\
M & S\end{array}$ & $\begin{array}{r}0 \\
1 \\
\text { II } \\
\text { III } \\
\text { IV }\end{array}$ & \begin{tabular}{|rr|r|}
$90 / 90^{\prime}$ & 1 \\
21 & 180 \\
209 & 114 & 1 \\
73 & 42 & 1 \\
35 & 26 & 1 \\
\end{tabular} & $\begin{array}{l}1.19 \\
1.16 \\
1.13 \\
1.13 \\
\end{array}$ & $\begin{array}{l}0.23 \\
0.33 \\
0.33 \\
1\end{array}$ & 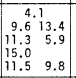 & $\begin{array}{|cc|} & 57.2 \\
51.6 & 56.6 \\
62.2 & 49.9 \\
57.6 & \\
55.7 & 46.7\end{array}$ & 0.58 & & $\begin{array}{ll}20 . & \\
& 55 . \mathrm{f} . \\
M & \mathrm{I}\end{array}$ & \begin{tabular}{r|}
0 \\
II \\
II \\
IV \\
\end{tabular} & $\begin{array}{|cc|}43 / 120 \\
6 & 87 \\
390 & 431 \\
437 & 324 \\
147 & 200 \\
\end{array}$ & $\begin{array}{l}1.86 \\
1.77 \\
1.73 \\
\end{array}$ & \begin{tabular}{|l|}
$0.6 \mathrm{C}$ \\
4.84 \\
7.64 \\
\end{tabular} & $\begin{array}{l}3.1 \\
1.4\end{array}$ \\
\hline $\begin{array}{l}\text { 5. } 20 . \mathrm{f} . \\
\text { A I }\end{array}$ & \begin{tabular}{r|rr|r}
0 & $56 / 1288$ & \\
I & 63 & 480 \\
II & 473 & 263 & 1 \\
II & 139 & 85 & 1 \\
IV & 33 & 22 & .
\end{tabular} & $\begin{array}{l}1.66 \\
1.44 \\
1.44 \\
1.46\end{array}$ & \begin{tabular}{|c|c|}
0.65 \\
0.97 & 1.06 \\
3.43 & 1.85 \\
3.87 \\
4.20
\end{tabular} & \begin{tabular}{|c|}
2.6 \\
17.1 \\
18.920 .1 \\
18.3 \\
14.315 .2 \\
10.0
\end{tabular} & \begin{tabular}{|c|}
39.1 \\
55.1 \\
23.7 \\
23.4 \\
72.4 \\
51.6 \\
51.6
\end{tabular} & $\begin{array}{l}\text { 13. } \\
61 . \mathrm{m} . \\
\text { Pericardial } \\
\text { effusion }\end{array}$ & \begin{tabular}{r|}
0 \\
1 \\
11 \\
111 \\
11
\end{tabular} & \begin{tabular}{|c|c|c|}
$40 / 120$ & 2 \\
36 & 230 & 2 \\
305 & 230 & 2 \\
185 & 165 & 2 \\
105 & 85 & 2 \\
105 & 8
\end{tabular} & $\mid$\begin{tabular}{l|l}
2.02 & 0 \\
2.03 & 0 \\
2.10 & 0 \\
2.13 & \\
&
\end{tabular} & $\begin{array}{l}0.17 \\
0.17 \\
0.09 \\
\end{array}$ & \begin{tabular}{|r|r|}
12.5 & \\
6.0 & 8.0 \\
9.9 & 13.4 \\
8.0 & 13.1 \\
16.8 & 15.7
\end{tabular} & \begin{tabular}{r|r|}
283.1 & \\
286.2 & 250.5 \\
180.3 & 118.2 \\
205.2 & 266.4 \\
260.7 & 280.2
\end{tabular} & \begin{tabular}{|l|}
6.38 \\
2.07 \\
3.09 \\
\end{tabular} & & $\begin{array}{l}\text { 21. } \\
\text { M S I, A I }\end{array}$ & $\begin{array}{r}0 \\
1 \\
11 \\
11 \\
\text { II } \\
\text { IV }\end{array}$ & 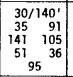 & \begin{tabular}{|l|}
2.70 \\
1.98
\end{tabular} & $\begin{array}{c}1.16 \\
10.5 \\
6.07\end{array}$ & $\begin{array}{l}2.0 \\
2.3\end{array}$ \\
\hline $\begin{array}{l}\text { 6. } 58 . \mathrm{m} \\
\text { I H o, } \mathrm{mi} \\
\text { Pheochromo- } \\
\text { cytoma }\end{array}$ & 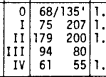 & $\begin{array}{l}1.77 \\
1.79 \\
1.66 \\
1.74\end{array}$ & 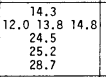 & & & $\begin{array}{l}\text { 14. } 44 . \mathrm{m} . \\
\text { Constrictive } \\
\text { pericarditis }\end{array}$ & $\begin{aligned} \\
1 \\
\text { II } \\
\text { III } \\
\text { IV } \\
\end{aligned}$ & \begin{tabular}{|c|c|}
$60 / 120^{\prime}$ & 2 \\
20 & 84 \\
207 & 213 \\
209 & 148 \\
189 & 148 \\
134 & 109 \\
\end{tabular} & \begin{tabular}{|l|l}
2.03 & 0 \\
2.03 & 2 \\
2.06 & 1 \\
\end{tabular} & $\begin{array}{c}0.50 \\
2.50 \\
1.85\end{array}$ & 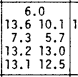 & 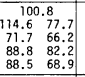 & $\begin{array}{l}0.93 \\
1.25 \\
1.01\end{array}$ & & 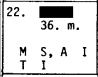 & \begin{tabular}{r|}
0 \\
1 \\
II \\
III \\
IV \\
\end{tabular} & 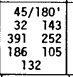 & 1.51 & $\begin{array}{l}0.21 \\
3.61 \\
2.20\end{array}$ & $\begin{array}{r}<2.2 \\
5.5\end{array}$ \\
\hline $\begin{array}{l}\text { 7. } 70 . \mathrm{f} \text {. } \\
\text { AS D }\end{array}$ & \begin{tabular}{r|rr|r}
0 & $42 / 120$ & 1. \\
II & 21 & 147 \\
II & 238 & 215 \\
III & 181 & 161 \\
IV & 129 & 103 & 1. \\
\end{tabular} & $\begin{array}{l}1.73 \\
1.71 \\
1.77 \\
1.73\end{array}$ & 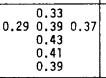 & $\begin{array}{|cc|}1.5 \\
6.2 & 11.2 \\
6.1 & 8.5 \\
6.5 & 11.5 \\
4.7 & 8.3 \\
\end{array}$ & 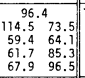 & $\begin{array}{l}15 . \quad 19 . \mathrm{m} . \\
\text { Control }\end{array}$ & $\begin{array}{r}0 \\
\text { I } \\
\text { II } \\
\text { III } \\
\text { IV }\end{array}$ & $\left|\begin{array}{rr|}120 / 155 & 1 \\
80 & 377 \\
472 & 231 \\
116 & 76 \\
35 & 25\end{array}\right|$ & $\begin{array}{l}1.75 \\
1.37\end{array}$ & & $\begin{array}{l}0.99 \\
3.12 \\
3.52\end{array}$ & $\begin{array}{l}2.06 \\
5.30 \\
3.97\end{array}$ & $\begin{array}{l}3.46 \\
3.36\end{array}$ & $\begin{array}{l}0.7 \\
2.6 \\
5.1\end{array}$ & $\begin{array}{l}23 . \\
27 . \mathrm{m} . \\
\text { MS I, A I }\end{array}$ & \begin{tabular}{|r|}
0 \\
I \\
III \\
II \\
\end{tabular} & \begin{tabular}{|c|c|}
$43 / 120$ \\
13 & 153 \\
245 & 187 \\
125 & 85 \\
76 & 62 \\
\end{tabular} & $\begin{array}{l}1.95 \\
1.60 \\
1.68 \\
\end{array}$ & & $\begin{array}{l}3.8 \\
2.8\end{array}$ \\
\hline $\begin{array}{l}8 . \quad 39 . \mathrm{m} . \\
\text { Constrictive } \\
\text { pericarditis }\end{array}$ & 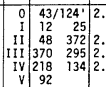 & \begin{tabular}{l|}
2.47 \\
2.19 \\
2.35 \\
2.16
\end{tabular} & \begin{tabular}{|r|}
1.12 \\
1.48 \\
1.68 \\
5.87 \\
4.53 \\
3.73 \\
3.26 \\
\end{tabular} & \begin{tabular}{|c|c|}
11.9 \\
12.9 \\
10.8 \\
5.9 .4 \\
9.9 & 5.0 \\
9.1 & 6.6 \\
10.3 &
\end{tabular} & 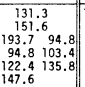 & $\begin{array}{l}16.21 . \mathrm{m} . \\
\text { Control }\end{array}$ & $\begin{aligned} 0 \\
I \\
I 1 \\
\text { III } \\
\text { IV }\end{aligned}$ & 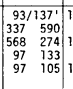 & $\begin{array}{l}1.74 \\
1.48 \\
1.41\end{array}$ & & \begin{tabular}{|c|c|}
0.26 \\
1.44 & 1.15 \\
1.922 & 1.20 \\
6.08 & 3.42 \\
3.13 & 2.24
\end{tabular} & \begin{tabular}{lc|}
\multicolumn{3}{c|}{7.18} \\
7.86 & 4.16 \\
6.48 & 6.05 \\
9.70 & 6.86 \\
6.74 & 8.34
\end{tabular} & & $\begin{array}{l}2.1 \\
4.7\end{array}$ & 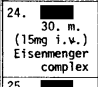 & \begin{tabular}{|r|}
0 \\
I \\
III \\
II \\
\end{tabular} & $\begin{array}{|cc|}19 / 1251 \\
66 & 61 \\
41 & 58 \\
& 81 \\
& \\
\end{array}$ & $\begin{array}{l}1.51^{*} \\
1.28 \\
1.13\end{array}$ & & 3.3 \\
\hline \multirow{4}{*}{$\begin{array}{l}\text { UV: urine } \\
\text { ALDo: uring } \\
\text { pertiod, II } \\
\text { steno-insu } \\
\text { TI: tricus } \\
\end{array}$} & & & & & & & & & & & & & & & 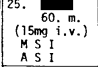 & $\begin{array}{r}0 \\
\text { I } \\
\text { III }\end{array}$ & 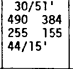 & $\begin{array}{l}1.80 \\
1.58 \\
1.44\end{array}$ & & $\begin{array}{l}1.6 \\
.3\end{array}$ \\
\hline & & & & & & & & & & & & & & & $\begin{array}{c}\text { 26. } 29 . \mathrm{f} \text {. } \\
\text { Control }\end{array}$ & \begin{tabular}{r|}
0 \\
II \\
III \\
IV
\end{tabular} & $\begin{array}{|cc|}48 / 30^{0} \\
22 & 96 \\
45 & 33 \\
376 & 528 \\
274 & 158 \\
\end{array}$ & $\begin{array}{l}2.10 \\
2.18 \\
1.66 \\
1.69\end{array}$ & & $\begin{array}{l}1.2 \\
6.8\end{array}$ \\
\hline & & & & & & & & & & & & & & & $\begin{array}{l}\text { 27. } \\
\text { Control }\end{array}$ & \begin{tabular}{r|}
0 \\
$I$ \\
II \\
III \\
IV
\end{tabular} & $\mid$\begin{tabular}{cc|}
$24 / 120^{\circ}$ \\
13 & 13 \\
10 & 13 \\
16 & 22 \\
366 & 299
\end{tabular} & $\begin{array}{l}1.47 \\
1.51 \\
0.99 \\
\end{array}$ & $\begin{array}{l}0.34 \\
0.57 \\
2.01\end{array}$ & $\begin{array}{l}1.4 \\
1.2\end{array}$ \\
\hline & & & & & & & & & & & & & & & $\begin{array}{l}\text { 28. } 55 . \mathrm{f} \text {. } \\
\text { Control }\end{array}$ & \begin{tabular}{r|}
0 \\
II \\
II \\
II
\end{tabular} & $\mid$\begin{tabular}{c|c|}
$100 / 128^{\circ}$ \\
79 & 634 \\
29.9 & 200 \\
115 & 42 \\
172 & 42
\end{tabular} & $\begin{array}{l}1.61 \\
1.31 \\
1.12 \\
\end{array}$ & & $\begin{array}{r}4.5 \\
2.3 \\
200 .\end{array}$ \\
\hline
\end{tabular}

of detailed combined evaluations of the responses of E, NE and PRA to furosemide. Although E and PRA changed together in 9 out of 13 cases and the increase of $\mathrm{E}$ appeared to precede that of PRA, the sequence of events is not clear. No clear-out relationship was seen between $\mathrm{E}$ and aldosterone, or PRA and aldosterone. Our previous investigations and the present study show that the increase in urinary $\mathrm{NE}$ is remarkable in $\mathrm{CHF}$, while $\mathrm{E}$ changes markedly in response to furosemide-induced acute diuresis. The explanation for this must await further investigations.

The possibility is proposed that the renin-angiotensin system stimulates the secretion of aldosterone on one occasion, and the release of $\mathrm{ADH}$ on another, although the selecting or switching mechanism is not known.

\section{Conclusion}

In chronic congestive heart failure, aldosterone and $\mathrm{ADH}$ act physiologically to maintain the adequate circulating blood volume through the regulation of sodium and water, respectively. Catecholamines act to regulate the cardiovascular tone to maintain proper circulation. Renin seems to act as a mediator among the factors, not only in the renin-angiotensin-aldosterone system, but also in the renin-angiotensin$\mathrm{ADH}$ system, as well as by interaction with catecholamines. 


\section{Acknowledgment}

We wish to pay tribute to the memory of the late Professor Emeritus M. Maekawa, who introduced us to this field of study. We thank Prof. S. Hayase and M. Miyahara, chairmen, for giving us the opportunity to join the symposium, and gratefully acknowledge the co-operation of our colleagues in Kyoto University, especially Drs. Y. Ohara, S. Kubo and K. Bito.

\section{References}

1) Maekawa, M.: J. Jap. Soc. Intern. Med. 53: 911, 1964.

2) Saimyoji, H. et al.: Proc. Third Asian-Pacific Congr. Cardiol., Kyoto, p. 1386, 1964.

3) Nohara, Y., Saimyoji, H.: Jap. J. Med. 4: 279, 1965 \& J. Jap. Soc. Intern. Med. 53 : 1255, 1964.

4) Maekawa, M., Saimyoji, H.: Jap. Circul. J. 30: 42, 1966.

5) Saimyoji, H., Ohara, Y.: ibid. 30: 1127, 1966.

6) Yasui, H.: ibid. 32: 467, 1968.

7) Kubo, S. : ibid. 35: 1183, 1971.

8) Takayasu, M.: Proc. 17th Jap. Med. Congr. 4: 333, 1967.

9) Saimyoji, H.: Jap. Circul. J. $33: 778,1969$.

10) Saimyoji, H., Seriu, Y., Kubo, S. : J. Clin. Sci. 6: 1569, 1970.

11) Rosenthal, J. et al.: Can. J. Physiol. Pharmacol. 46: 85, 1968.

12) Espiner, E. A. et al.: New Engl. J. Med. 280: 1141, 1969.

13) Brown, T.C. et al.: Am. J. Physiol. 211: 437, 1966.

14) Meyer, P. et a1.: Rev. Can. Biol. 25: 111, 1966.

15) Meyer, P. et al.: Am. J. Physiol. 215: 908, 1968. 